\title{
Critical analysis of the use of onabotulinumtoxinA (botulinum toxin type $A$ ) in migraine
}

This article was published in the following Dove Press journal:

Neuropsychiatric Disease and Treatment

12 January 2012

Number of times this article has been viewed

\section{Carrie E Robertson Ivan Garza}

Department of Neurology, Mayo Clinic, Rochester, MN, USA

Correspondence: Carrie E Robertson Department of Neurology, 200 First Street SW, Mayo Clinic, Rochester, MN, USA

Tel + I 507538 I036

Fax +I 5072664419

Email carrie.robertson@mayo.edu
Abstract: OnabotulinumtoxinA, a neurotoxin, has been studied in numerous trials as a novel preventive therapy for migraine headache. The data would support that it may be effective at reducing headache days in patients suffering from chronic migraine ( $\geq 15$ headache days/month, with eight or more of those migraine headache days). The mechanism by which onabotulinumtoxinA exerts its effects on migraine is not yet understood. It is known to inhibit acetylcholine release at the neuromuscular junction, but this probably does not explain the observed antinociceptive properties noted in preclinical and clinical trials. This review will discuss the known mechanisms of action of botulinum toxin type A, and will review the available randomized, placebo-controlled trials that have looked at its efficacy as a migraine preventative. We also describe the onabotulinumtoxinA injection sites used at our institution.

Keywords: botulinum toxin, prophylaxis, onabotulinumtoxinA, mechanism, migraine, prevention

\section{Introduction}

Migraine is a debilitating primary headache disorder characterized by recurrent headaches typically described as unilateral, pulsating, moderate, or severe. They are aggravated by physical activity and associated with nausea and/or sensitivity to light and sound. ${ }^{1}$ Though it is often conceptualized as a continuum or spectrum disorder, migraine is divided into two subtypes based on attack frequency: episodic and chronic. In episodic migraine, attacks occur on less than 15 days each month, whereas in chronic migraine a patient has at least 15 headache days each month, and at least eight of those are migraine headaches (see Table 1). ${ }^{2,3}$ Each year, $3 \%-14 \%$ of episodic headache sufferers convert to chronic headache sufferers. ${ }^{1}$ Compared with episodic migraine sufferers, patients with chronic migraine have a lower socioeconomic status, reduced health-related quality of life, greater psychiatric and medical comorbidities, and increased occupational disability. ${ }^{4}$

The main aim of preventive migraine therapy is to reduce the frequency, duration, and/or severity of migraine attacks. There are many preventive therapies available, including $\beta$-adrenergic blockers, antidepressants, calcium channel blockers, and anticonvulsants. ${ }^{5}$ Unfortunately, many of the current options are of limited benefit and can be associated with potentially serious side effects. ${ }^{6}$ There is therefore great demand for alternative preventive therapies that are effective and well tolerated, with limited systemic effects. ${ }^{6}$

Botulinum toxin type A (BT-A) was first reported as a potential migraine therapy by Binder et $\mathrm{al}^{7}$ in 1991, when they observed that the patients receiving pericranial 
Table I Criteria for migraine without aura and chronic migraine (from the International Headache Society) ${ }^{2,3}$

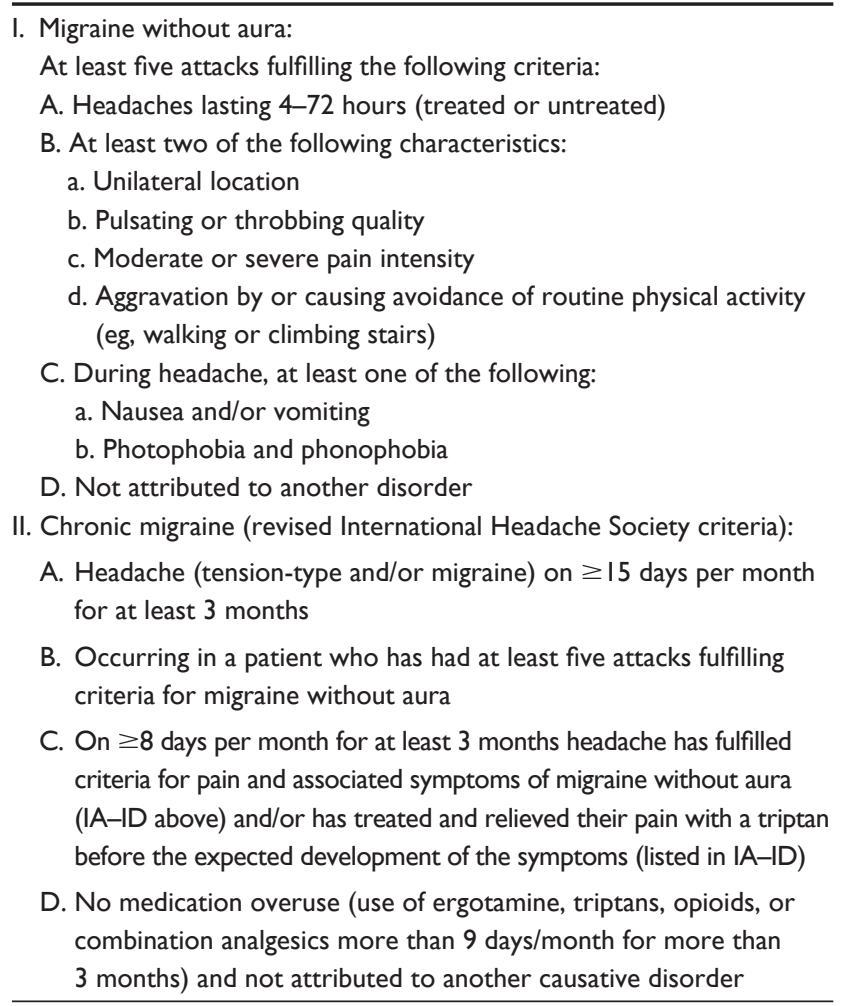

BT-A injections for facial hyperfunctional lines experienced relief of migraine headache symptoms. A nonrandomized, open-label study suggesting that BT-A could possibly be a safe and effective migraine treatment soon followed. ${ }^{7,8}$ Since then, great interest has prevailed in this pharmaceutical as a potential treatment for migraine. While investigating its efficacy as a migraine preventive, there have also been efforts to establish the mechanism by which botulinum toxin might exert an analgesic effect.

The goal of this review is to discuss the known mechanisms of action of BT-A, and then discuss the evidence behind its use in the treatment of migraine. As pointed out by Dressler and Benecke ${ }^{9}$ in a recent review, there is some discrepancy in the abbreviations used in the literature for these types of discussions. For our purposes, we will use the neurotoxin abbreviation (BoNT-A) for discussion of mechanism of action at the level of the neuromuscular junction, and then use the therapeutic preparation botulinum toxin type A abbreviation (BT-A) for discussions of preclinical and clinical trials. At the time of this article, only BT-A in the form of onabotulinumtoxinA has been approved by the US Food and Drug Administration (FDA) for the treatment of chronic migraine. Therefore, our discussion of clinical efficacy will focus primarily on published data from randomized, double-blind, placebo-controlled trials examining the use of onabotulinumtoxinA in migraine prophylaxis.

\section{Pharmacology of botulinum toxin}

Strains from the gram-positive anaerobic bacteria Clostridium botulinum are known to produce seven serologically distinct neurotoxins: A, B, C1, D, E, F, and G (C2 is not considered a neurotoxin). ${ }^{10}$ Currently, only the $\mathrm{A}$ and $\mathrm{B}$ serotypes are used for commercial purposes, though others have been used experimentally in humans. ${ }^{11,12}$ Because of differences in production, the commercially available botulinum toxin preparations each have distinct pharmacokinetics. The potency units are specific to each product, and the doses cannot be compared or converted from one botulinum product to another. To reinforce these individual potencies and prevent medication errors, the FDA has revised the classification of botulinum toxin products to include the following generic names: onabotulinumtoxinA (marketed as BOTOX ${ }^{\circledR} /$ BOTOX $^{\circledR}$ Cosmetic, Allergan Inc, Irvine, CA), rimabotulinumtoxinB (MYOBLOC ${ }^{\circledR}$, Solstice Neurosciences Inc, Louisville, KY), abobotulinumtoxinA (Dysport ${ }^{\circledR}$, Medicis Pharmaceuticals, Scottsdale, AZ), and incobotulinumtoxinA $\left(\right.$ XEOMIN $^{\circledR}$, Merz Pharmaceuticals, Greensboro, NC). ${ }^{13,14}$

Therapeutic preparations of BT-A come as powders that have to be reconstituted with $0.9 \% \mathrm{NaCl} / \mathrm{H}_{2} \mathrm{O}$ before use. Most BT-A preparations consist of the botulinum toxin (neurotoxin and nontoxic complexing proteins) and excipients. ${ }^{9}$ IncobotulinumtoxinA is slightly different, in that it has no complexing proteins. ${ }^{15}$ With food-borne botulism, the complexing proteins are thought to help protect the protein from acids and proteases in the gastrointestinal tract, and may help the toxin cross the intestinal barrier to enter circulation. The role of complexing proteins in commercial preparations of injectable botulinum toxin is not clear. ${ }^{15}$ The active neurotoxin molecule is made up of a heavy chain $(100 \mathrm{kDa})$ and a light chain $(50 \mathrm{kDa})$, joined by a disulfide bond (see Figure 1). The heavy chain acts as the binding and translocation domain, and the light chain is the enzymatically active zinc $\left(\mathrm{Zn}^{++}\right)$-dependent endopeptidase. ${ }^{16,17}$

\section{Method of action}

\section{Action at the neuromuscular junction}

The most well-known mechanism of action is at the presynaptic nerve terminal, where the toxin prevents the calciumdependent release of acetylcholine. ${ }^{18}$ There are four main steps in this process: binding to the acceptor on the plasma membrane, internalization of the botulinum toxin, separation of the light and heavy chain with escape of the light chain 

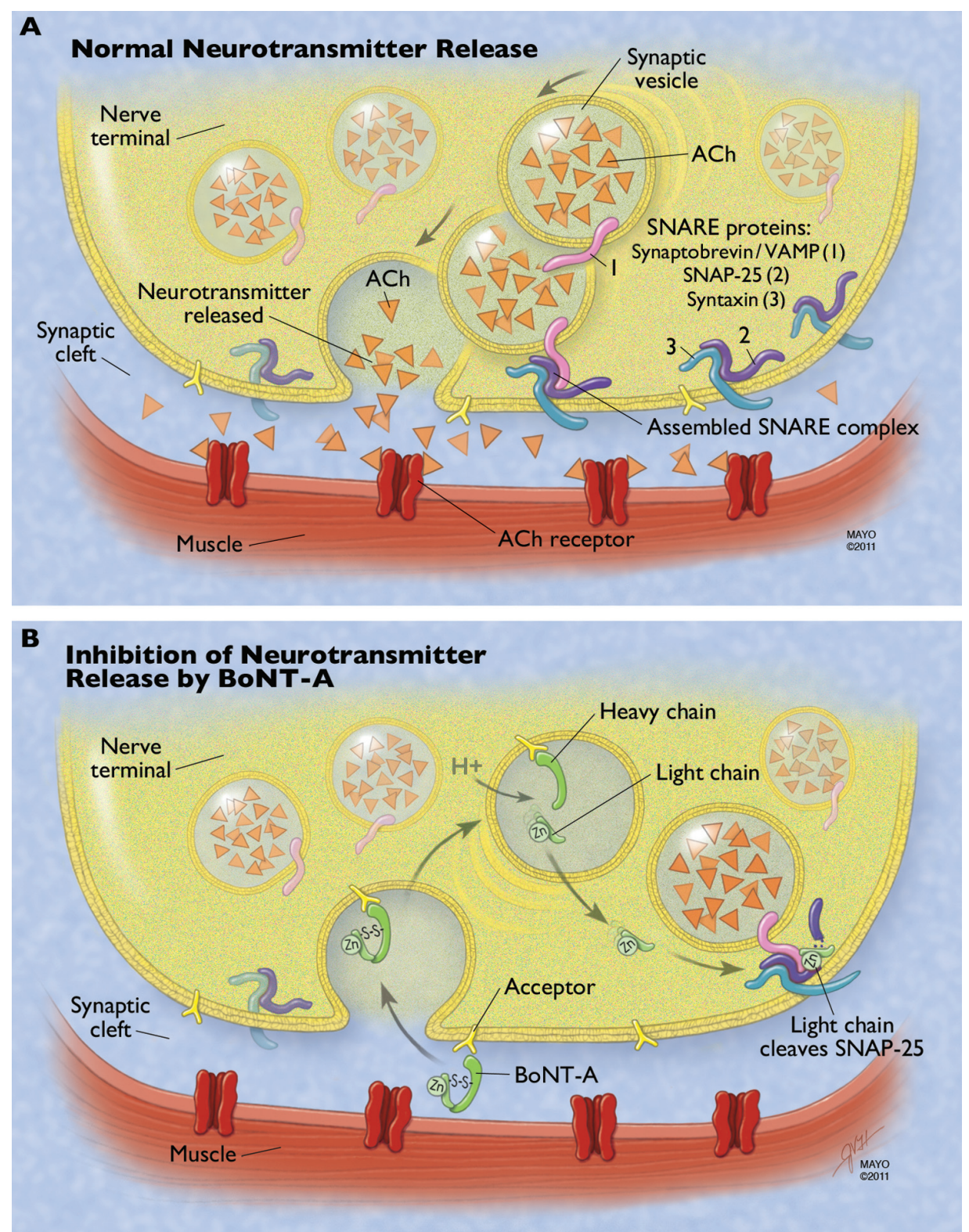

Figure I Mechanism of botulinum neurotoxin type A (BoNT-A) at the neuromuscular junction. (A) Normal neurotransmitter release requires fusion of the vesicle membrane to the membrane of the presynaptic nerve terminal. This process is guided by the fusion of three proteins that make up the soluble $\mathrm{N}$-ethylmaleimide-sensitive factor attachment protein receptor (SNARE) complex: the vesicle-associated membrane protein (VAMP or synaptobrevin) and the membrane-bound syntaxin and synaptosomeassociated protein of $25 \mathrm{kDa}$ (SNAP-25). ACh is released from the vesicle, diffuses across the synaptic cleft, and binds to the acetylcholine (Ach) receptor, resulting in normal muscular contraction. (B) The heavy chain of BoNT-A binds to a ganglioside acceptor in the plasma membrane of the presynaptic nerve terminal. This leads to receptormediated endocytosis of the neurotoxin. The acidic environment of the synaptic vesicle or endosome leads to a conformational change in the toxin and eventual reduction of the linking disulfide bond, freeing the light chain. The light chain translocates to the cytosol and cleaves the C-terminal of the SNAP-25 protein. This inhibits SNARE complex formation and therefore inhibits neurotransmitter release.

Note: Used with permission of Mayo Foundation for Medical Education and Research, all rights reserved.

into the cytosol, and light-chain inhibition of the docking/ fusion of the synaptic vesicles containing acetylcholine. ${ }^{19}$ These steps are illustrated in Figure 1 and are described in further detail in this section.

The C-terminal of the heavy chain is responsible for interacting with a high-affinity ganglioside acceptor outside of the nerve terminal. ${ }^{18}$ This acceptor is felt to be an intraluminal vesicular protein that has become exposed to the outside of the nerve terminal as part of the recycling/exocytosis of vesicles. ${ }^{18}$ This induces endocytosis of the botulinum neurotoxin (BoNT) into the nerve terminal, where it is contained within a membrane-bound synaptic vesicle. The fact that BoNT may depend on the recycling of vesicles to gain entry has been proposed as an explanation for why BoNT seems to be more effective in blocking neuromuscular junctions when the target muscle is active. ${ }^{17,18,20,21} \mathrm{Next}$, the acidic 
environment of the vesicle induces a conformational change of the toxin, with eventual breaking of the disulfide bond. This allows the light chain to escape the vesicle into the cytosol. ${ }^{18,22}$ The light chain then cleaves one of three core proteins that comprise the soluble N-ethylmaleimide-sensitive factor attachment protein receptor (SNARE) complex and that are involved in vesicle docking/fusion during regulated exocytosis. The three core SNARE proteins include vesicle-associated membrane protein (VAMP; also known as synaptobrevin), syntaxin, and synaptosomal-associated protein of $25 \mathrm{kDa}$ (SNAP-25). ${ }^{23}$ Each immunologically distinct BoNT serotype has a unique site of action within the SNARE complex (BoNT-C1 actually has two sites of action). Synaptobrevin/VAMP is cleaved by BoNT types B, D, F, and G. Syntaxin is cleaved by BoNT type C1. SNAP-25 is cleaved by BoNT types A, E, and $\mathrm{C} 1 .{ }^{24}$ By inhibiting the release of acetylcholine, BoNT leads to chemical denervation and muscle paralysis. Recovery from toxin-induced paralysis involves resprouting of new terminals from the motor neuron, followed by slow recovery of the original nerve terminal's ability to release acetylcholine. ${ }^{25}$ For BoNT-A, the clinical effect terminates in about 2-6 months in humans. ${ }^{26}$

\section{Antinociceptive action}

Early in the use of botulinum toxin for motor conditions, investigators noted that the pain relief preceded, and in some cases was greater than, the objective motor benefit; suggesting that the effect on pain probably involved more than just neuromuscular blockade. ${ }^{19,27}$ The antinociceptive action of BT-A, and the effect on migraine, is poorly understood; but the effect may be multifactorial with effects on muscle fibers, autonomic fibers, and possibly pain fibers. BT-A appears to have an effect on peripheral sensitization and may also have an effect (either direct or indirect) on the central processing of pain..$^{27,28}$

\section{Action at other cholinergic nerve terminals}

The neuromuscular blockade just described is a result of blocking the $\alpha$-motor neurons innervating the extrafusal muscle fibers, which are the fibers responsible for the actual contractile property of the muscle. However, BT-A is also known to block transmission of $\gamma$-motor neurons to the intrafusal fibers in muscle spindles. ${ }^{17}$ As muscle spindles provide afferent information about muscle stretch to the central nervous system (CNS), some authors have suggested that reducing their input may attenuate the hyperactivity of the muscle involved in tonic muscle contractions, and may in this way contribute to some of the pain relief observed. ${ }^{29,30}$
In addition to the somatic motor neurons ( $\alpha$ and $\gamma$ ), BT-A interferes with transmission of acetylcholine in the autonomic nervous system. By blocking the preganglionic nerve terminals of the sympathetic and parasympathetic nerve terminals, as well as many of the postganglionic fibers, botulinum toxin has a complex effect on autonomic function. In botulism, this effect can lead to symptoms of anhidrosis, xerophthalmia, xerostomia, orthostatic hypotension, gastrointestinal paralysis, and urinary retention. Therapeutically, this effect has made botulinum toxin a useful therapy for hyperhidrosis and hypersalivation. Many of the autonomic nerve terminals affected by botulinum toxin have other neuropeptides colocalized with acetylcholine, such as substance P, somatostatin, enkephalins, norepinephrine, adenosine triphosphate, neuropeptide $\mathrm{Y}$, and nitric oxide. ${ }^{30}$ Some authors hypothesize that the effect of BT-A on the autonomic nervous system, particularly the effect on autonomic vascular control, may allow the toxin to interfere with neurogenic inflammation and associated pain. ${ }^{30}$

\section{Noncholinergic nerve terminals}

In vitro and in vivo studies have demonstrated that if BT-A is introduced into noncholinergic nerve terminals, it can block the calcium-dependent release of neurotransmitters other than acetylcholine. ${ }^{17,27,28}$ BT-A has been found to block the release of inflammatory pain mediators such as substance $\mathrm{P}$ from cultured dorsal root ganglion neurons. It has also been shown to reduce potassium-stimulated, but not basal, release of calcitonin gene-related peptide (CGRP) from cultured trigeminal ganglia neurons. ${ }^{31,32}$ It has also been shown to block the release of glutamate from peripheral nerve terminals in inflammatory pain models. ${ }^{33}$

\section{Inflammatory and neuropathic pain models}

In rat models of inflammatory pain, peripherally applied BT-A has been shown to be effective in reducing the associated pain behaviors. Cui et $\mathrm{al}^{33}$ examined the effect of BT-A on the formalin model of inflammatory pain. In this model, a chemical irritant (formalin) is injected into the hind paw of a rat. This results in a reproducible biphasic response involving pain behaviors and excitation of dorsal horn neurons. Phase I (first 5 minutes after injection) is thought to be caused by direct activation of the peripheral small afferent fibers by formalin. Following a quiet phase, phase II (15-60 minutes) is attributed to sensitization of the peripheral afferents by inflammatory mediators. Pretreatment with BT-A was shown to produce a dose-dependent reduction in nociceptive behaviors during phase II. ${ }^{33}$ 
Further study showed that this reduction in pain behavior was associated with a dose-dependent reduction of the excitability of the dorsal horn (as measured by Fos-like immunoreactivity) and of the formalin-mediated activity in the dorsal horn wide-dynamic-range neurons. There was no effect on acute pain behavior during phase I, which agrees with previous studies showing that BT-A has no direct effect on acute noninflammatory nociception. ${ }^{27,34}$ In other inflammatory pain models, BT-A pretreatment ( 6 days) also significantly reduced the enhanced sensitivity to mechanical and thermal stimuli provoked by peripheral carrageenan or capsaicin injections in rats. ${ }^{35}$

Animal models have also examined the effect of BT-A in neuropathic pain models. Bach-Rojecky et $\mathrm{al}^{36}$ demonstrated that a peripheral injection of BT-A significantly reduced thermal and mechanical hyperalgesia associated with the neuropathy induced by partial sciatic nerve transection in rats. Similar to other studies, there was no direct antinociceptive effect, as the BT-A had no effect on the thermal and mechanical sensation of sham-operated animals. ${ }^{36}$ Pavone and Luvisetto ${ }^{28}$ also found a dose-dependent reduction in mechanical allodynia and cold hyperalgesia following chronic constriction injury of the sciatic nerve. The effect on allodynia was present only if BT-A was given after nerve injury, suggesting that BT-A could reduce neuropathic symptoms but not protect against the neuropathy. A similar effect was present if the BT-A was injected intrathecally. ${ }^{37}$ Not only does BT-A affect pain behaviors associated with chronic constriction injury of the sciatic nerve but also it was found to diminish the expected injury-induced upregulation of neuropeptides (eg, prodynorphin and pronociceptin) and nitric oxide synthase $1 \mathrm{mRNA}$ in the rat dorsal root ganglia. ${ }^{38}$

Human trials may also support BT-A having an analgesic effect in neuropathic pain. In a recent study, Ranoux et $\mathrm{al}^{39}$ used a randomized, double-blind, placebo-controlled trial to examine the effect of BT-A on allodynia associated with focal neuropathies. They found that a single intradermal injection of BT-A reduced the intensity and area of mechanical allodynia and cold pain thresholds on the affected side, with no change in perception thresholds. This effect seemed to persist from 2 weeks to 14 weeks after injection.

\section{Possible central action of BT-A}

While studying the effect of BT-A on paclitaxel-induced neuropathy in rats, Favre-Guilmard et $\mathrm{al}^{40}$ noted that injection of BT-A in the hindpaw almost completely abolished the mechanical hyperalgesia not only in the injected paw but also in the contralateral paw. This bilateral antinociceptive effect remained stable over the 6 days of observation. The mechanism behind this bilateral effect was unclear, but the authors pointed out that a systemic diffusion effect was unlikely, as a contralateral injection of BT-A did not elicit an antihyperalgesic effect in their model of carrageenan-induced hyperalgesia or in previous models of formalin-induced pain. ${ }^{40}$ They proposed that the mechanism might involve more complex processes such as central sensitization. Bach-Rojecky et $\mathrm{al}^{41}$ observed a similar bilateral antinociceptive effect while studying the effect of a single injection of BT-A on streptozotocin-induced diabetic neuropathy in rats. Other studies have found that peripheral injections of BT-A in the cranial region can affect associated brainstem nuclei. For instance, BT-A injected into the cat lateral rectus muscle can have dose-dependent structural and functional effects on the abducens nuclei, including a reduction in the firing rate. ${ }^{42}$ Using a rat model of infraorbital nerve constriction, Kitamura et $\mathrm{al}^{43}$ found that BT-A injected 3 days following nerve injury not only reduced mechanical allodynia but also reduced exaggerated neurotransmitter release from trigeminal ganglion neurons.

Given the high-affinity binding of botulinum toxin to the peripheral cholinergic nerve terminals, many authors have hypothesized that any central effect of botulinum toxin was indirect; either related to an alteration in sensory input from the muscle spindles or as an effect of denervation on CNS plasticity. ${ }^{41,44}$ The possibility that BT-A could reach the CNS had been suggested in the 1970s, but it was felt that the retrograde axonal transport system was so slow that the toxin was likely to be inactive before it reached the cell body. ${ }^{28}$ However, in the last few years, studies have shown that at high doses, at least some amount of catalytically active BoNT-A may undergo retrograde transport to the motor nuclei supplying that nerve terminal. ${ }^{45}$ As axonal transport to the CNS within motor neurons would not necessarily explain a reduction in pain, a more recent study looked for similar evidence of retrograde transport within sensory neurons. Matak et $\mathrm{al}^{46}$ used a rat model of formalin-induced facial pain and examined the effects of BT-A injected into the rat whisker pad or sensory trigeminal ganglion. They found that BT-A at either location reduced the phase II pain. When colchicine was given to block microtubule-dependent transport, the antinociceptive effect was prevented. They also found BT-A-truncated SNAP-25 in the medullary dorsal horn of the trigeminal nucleus caudalis 3 days following injection into the whisker pad.

\section{Summary}

The precise mechanism of BT-A as a migraine preventive is still unknown but remains an area of great interest and 
ongoing research. Based on the available in vitro and in vivo models, it has been proposed that BT-A inhibits the release of pain-related neurotransmitters and neuropeptides such as substance P, CGRP, and glutamate from the peripheral termini of primary trigeminal and cervical afferents. This reduces peripheral sensitization. Because central sensitization results from ongoing input from pain fibers, the inhibition of these peripheral signals indirectly inhibits central sensitization. In addition, peripherally injected BT-A may be retrogradely transported along axons of peripheral nerves, allowing inhibitory effects at the level of the dorsal root ganglion and dorsal horn. ${ }^{28}$ Whatever the mechanism of action, it is the analgesic effects and low systemic side effects observed in clinical trials that have led to the growing use of botulinum toxin for migraine headache.

\section{Clinical efficacy studies}

As mentioned previously, the present discussion analyzes data from randomized, double-blind, placebo-controlled studies published in the English literature researching onabotulinumtoxinA specifically in adult migraine prophylaxis (see Table 2). ${ }^{47-57}$ Although small case series have shown encouraging results using onabotulinumtoxinA in pediatric chronic migraine, ${ }^{58,59}$ to this date no placebo-controlled studies have been published in English literature.

\section{Episodic migraine}

In the first randomized, double-blind, placebo-controlled study, Silberstein et $\mathrm{al}^{47}$ attempted to confirm Binder et al's ${ }^{6}$ findings. Subjects having two to eight moderate-to-severe migraines per month (with or without aura) were randomized to placebo or onabotulinumtoxinA ( $25 \mathrm{U}$ or $75 \mathrm{U}$ ). Subjects received symmetrical injections into the frontalis, temporalis, corrugator, and procerus muscles. The mean migraine frequencies per month at baseline in the placebo, $25 \mathrm{U}$, and $75 \mathrm{U}$ treatment groups were 4.8, 4.3, and 4.0, respectively. The study's primary efficacy variable was met. There was a significantly greater reduction in the number of moderate-tosevere migraines in the $25 \mathrm{U}$ group than in the placebo group at Month 2 (placebo, $-0.37 ; 25 \mathrm{U}$ onabotulinumtoxinA, -1.57 ; $P=0.008$ ) and Month 3 (placebo, -0.98; $25 \mathrm{U}$ onabotulinumtoxinA, $-1.88 ; P=0.042$ ) after injection. For unclear reasons, the $75 \mathrm{U}$ dose did not perform as well as the $25 \mathrm{U}$ dose. The authors suspected that this was secondary to the lower migraine frequency at baseline in the $75 \mathrm{U}$ treatment group.

Evers et $\mathrm{al}^{48}$ used different onabotulinumtoxinA doses with a specific focus on different injection sites. Sixty subjects having two to eight migraine attacks were enrolled.
Subjects were treated with either $100 \mathrm{U}$ in the frontal and neck muscles or with $16 \mathrm{U}$ in the frontal muscles and placebo in the neck muscles, or with placebo in all muscles. The primary efficacy parameter was not met. The rate of patients with at least a $50 \%$ reduction of migraine frequency was $30 \%$ in the group receiving $100 \mathrm{U}, 30 \%$ in the group receiving $16 \mathrm{U}$, and $25 \%$ in the group receiving placebo $(P=0.921)$. Regarding secondary efficacy parameters, the only significant difference observed was in the sum score of all accompanying symptoms. In the $16 \mathrm{U}$ group, but not in the $100 \mathrm{U}$ group, the accompanying symptoms (photophobia, phonophobia, nausea, and vomiting) were significantly reduced by $29 \%$ in Month 3 compared with a reduction of $5 \%$ in the placebo group ( $P=0.048$; posthoc test). Similar to Silberstein et al's ${ }^{47}$ study, it was the low-dose treatment group in which Evers et $\mathrm{al}^{48}$ were able to observe a significant finding, leading them to consider the total dosage of onabotulinumtoxinA a less important variable than other parameters such as injection sites and patient selection.

In a series of three sequential randomized, double-blind studies, Elkind et $\mathrm{al}^{49}$ further explored onabotulinumtoxinA for migraine prophylaxis in subjects suffering four to eight moderate-to-severe migraines per month. In study I, patients were randomized to placebo or onabotulinumtoxinA into frontal, glabellar, and temporal muscle at a dose of $7.5 \mathrm{U}$, $25 \mathrm{U}$, or $50 \mathrm{U}$. In study II, completers who received placebo or onabotulinumtoxin $7.5 \mathrm{U}$ were randomized to receive two treatments of either $25 \mathrm{U}$ or $50 \mathrm{U}$ onabotulinumtoxinA, whereas patients who had received onabotulinumtoxinA $25 \mathrm{U}$ or $50 \mathrm{U}$ continued the same dose for two additional treatments. Completers entered study III, where they were randomized to placebo or continued treatment with $25 \mathrm{U}$ or 50 U. All treatments across the three studies were administered at 4 month intervals. The primary efficacy measure was not met. Migraine frequency was not different among treatment groups at any visit in any of the studies (assessed as change from baseline, all $P \geq 0.201$ ). There was no statistically significant effect of onabotulinumtoxinA at any time. When comparing their results with more encouraging results obtained in chronic daily headache $(\mathrm{CDH})$ trials (with many chronic migraine patients), ${ }^{60}$ Elkind et al ${ }^{49}$ suggested that perhaps those with a greater frequency of migraine attacks are more responsive to onabotulinumtoxinA than subjects with episodic migraine.

Aurora et $\mathrm{al}^{50}$ performed a phase II trial using the "follow-the-pain" injection protocol. Instead of having the onabotulinumtoxin A injection sites and doses already fixed (referred to as the "fixed-site, fixed-dose" approach), 


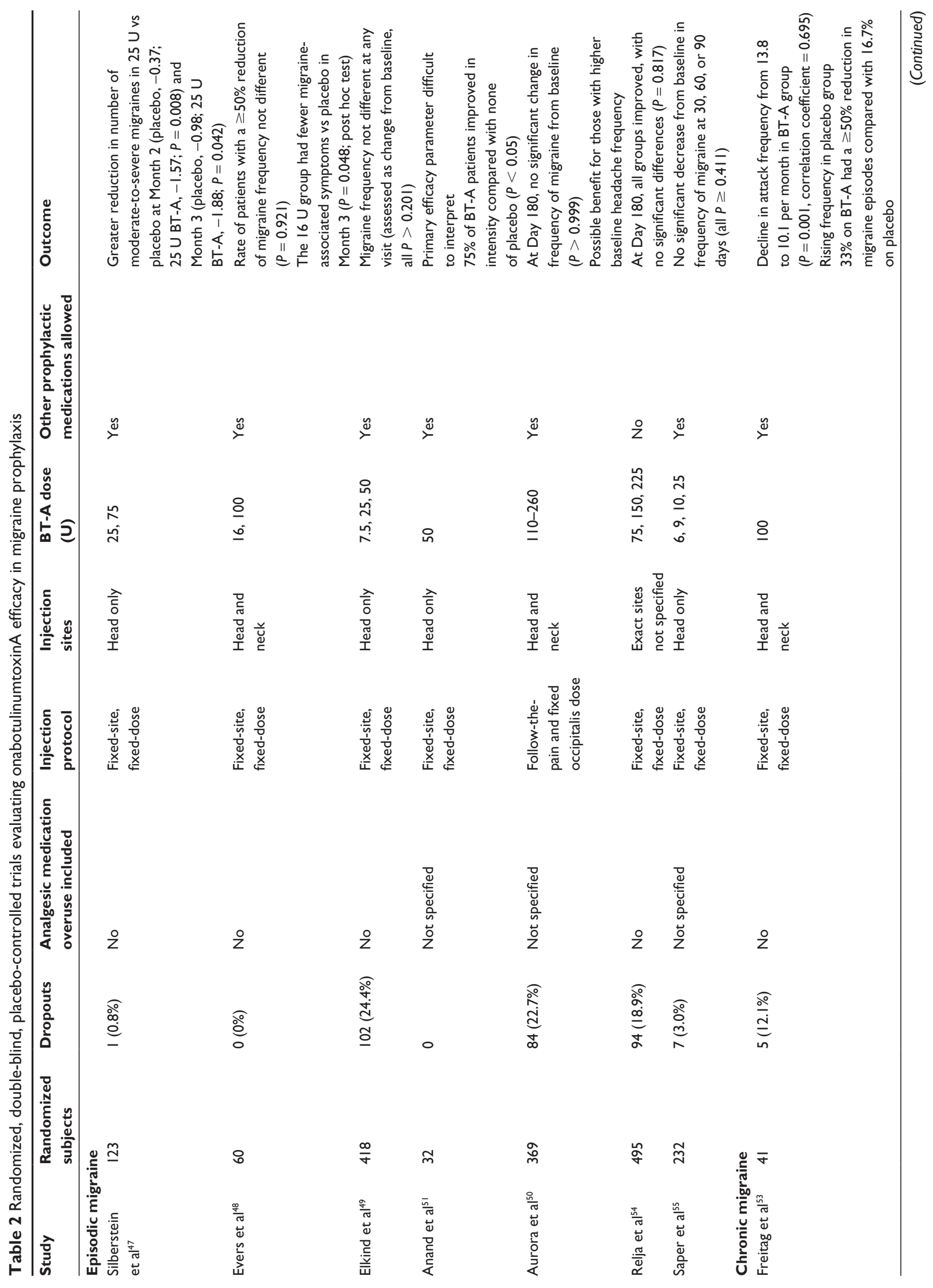




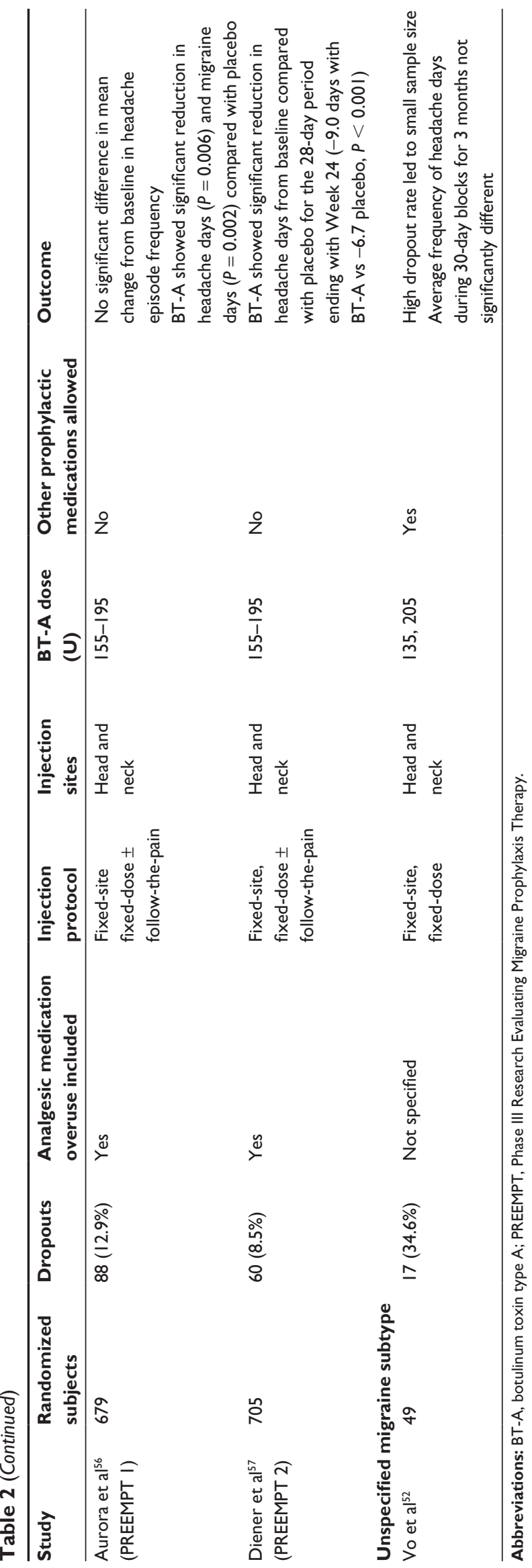

they allowed the investigator to define the injection sites and doses based on pain distribution and severity (except the occipitalis muscle, where dosing was fixed). A total of 369 subjects were randomized to three treatments with onabotulinumtoxinA $110-260 \mathrm{U}$ or placebo at 90 day intervals. OnabotulinumtoxinA did not show superiority over placebo in the primary efficacy measure. At Day 180, the mean change from baseline in migraine frequency per 30 day period was -2.4 in the onabotulinumtoxinA group compared with -2.2 in the placebo group $(P>0.999)$. There were no statistically significant differences between treatment groups. In a post hoc analysis, however, in the group of patients experiencing a higher baseline frequency ( $\geq 12$ headache days), patients receiving onabotulinumtoxinA experienced a significant reduction in migraine frequency, with a mean change from baseline of -4 headache episodes compared with -1.9 for the placebo patients $(P=0.048)$. Although the Day 180 time point was the only time point at which the between-group difference reached statistical significance, a consistent trend was observed within this subgroup of patients favoring onabotulinumtoxinA throughout the study.

In a small 3 month study, 32 subjects with four to eight migraine attacks per month were randomized to symmetrical injections of $50 \mathrm{U}$ of onabotulinumtoxinA in three pericranial muscle regions (frontalis, temporalis, and glabellar) versus placebo. ${ }^{51}$ The primary efficacy parameter was frequency of attacks per 4 weeks, and the effect on this was difficult to determine due to inconsistencies in the text of the manuscript. The secondary efficacy parameter was defined as severity of attacks, and the authors state that $75 \%$ of patients in the onabotulinumtoxinA group reported a marked improvement in the intensity of headaches from a "moderate-severe" category to "complete relief-mild" category, whereas none in the placebo group noted this improvement $(P<0.05)$.

Relja et $\mathrm{a}^{54}$ used a 30 day placebo run-in to divide 495 patients into placebo responders $(n=173)$ and placebo nonresponders $(n=322)$. They then injected $225 \mathrm{U}, 150 \mathrm{U}$, or $75 \mathrm{U}$ of onabotulinumtoxinA or placebo using a fixedsite, fixed-dose seven-site approach in pericranial and neck muscles. Patients received additional treatments at Day 90 and Day 180 and returned for follow-up visits at 30 day intervals following each treatment through Day 270. At Day 180 , the primary endpoint, the mean change from baseline in the frequency of migraine episodes in the placebo nonresponders stratum per 30 day period was $-1.6,-1.7,-1.5$, and -1.4 in the onabotulinumtoxinA $225 \mathrm{U}, 150 \mathrm{U}, 75 \mathrm{U}$, and placebo groups, respectively. The differences between the groups were not statistically significant $(P=0.817)$. 
A strong placebo response was evident in this trial. According to the authors, this could have been secondary to the uneven randomization scheme with a greater number of active treatment arms instead of an even 1:1 randomization.

Saper et $\mathrm{al}^{55}$ compared different injection sites and doses of onabotulinumtoxin $\mathrm{A}$ in patients with four to eight moderate-to-severe headaches per month. A total of 232 patients were randomized, with 45 assigned to placebo and 187 assigned to onabotulinumtoxinA $(n=44$ frontal, $\mathrm{n}=45$ temporal, $\mathrm{n}=49$ glabellar, $\mathrm{n}=49$ all three sites or "FTG"). The primary efficacy variable was frequency of migraine headaches, with Day 60 specified as the primary endpoint. Following intervention, baseline frequency ratings were 5.6 (FTG), 5.7 (frontal), 5.9 (temporal), 5.3 (glabellar), and 5.5 (placebo) migraine headaches per month $(P=0.399)$. No statistically significant among-group differences were observed for decreases from baseline in the frequency of migraines of any severity at the 30,60, or 90 day follow-up visit (all $P \geq 0.411$ ). According to the authors, low onabotulinumtoxinA dose, lack of posterior head and neck muscle injections, allowance of other preventive medication use, and exclusion of patients with chronic migraine may all have been reasons for a negative trial.

To conclude, although results from Silberstein et al's ${ }^{47}$ original study in episodic migraine were encouraging, the available data from randomized, double-blind, placebocontrolled studies do not convincingly show onabotulinumtoxinA to be effective in the prevention of episodic migraine. Based on the current evidence, a report of the Therapeutics and Technology Assessment Subcommittee of the American Academy of Neurology has labeled onabotulinumtoxinA as probably ineffective in episodic migraine treatment. ${ }^{61}$

\section{Chronic migraine}

In a small study from Freitag et al, ${ }^{53} 41$ patients were randomized to onabotulinumtoxinA $100 \mathrm{U}$ or placebo using a fixedsite, fixed-dose paradigm at the glabella, temporal, frontal, suboccipital, and trapezius muscles. Patients were excluded if they were overusing analgesics or caffeine. The primary efficacy parameter was the change in monthly migraine frequency per 4 week assessment period over 4 months compared with baseline. OnabotulinumtoxinA injections were found to be superior to placebo in terms of reduction of headache attack frequency, reducing headaches from 13.8 to 10.1 per month $(P=0.001$, correlation coefficient $=0.695)$, compared with the placebo arm that actually noted a rise in headache frequency from 14.6 to 15.4 per month $(P=0.046$, correlation coefficient $=0.475)$. In addition, six of $18(33 \%)$ completers on onabotulinumtoxinA had at least a $50 \%$ reduction in migraine episodes compared with three of 18 (16.7\%) placebo patients.

The PREEMPT 1 (Phase III Research Evaluating Migraine Prophylaxis Therapy-1) trial ${ }^{56}$ had a 24 week, double-blind, parallel-group, placebo-controlled phase followed by a 32 week, open-label phase. A total of 679 patients were randomized 1:1 to onabotulinumtoxinA or placebo injected every 12 weeks. Two cycles of injections were administered. Two-thirds of patients were overusing acute medications, though patients using frequent opiates were avoided at enrollment. No pharmacologic prophylactics were allowed. OnabotulinumtoxinA or placebo was administered using fixed-site, fixed-dose injections across seven head and neck muscle areas (corrugator, procerus, frontalis, temporalis, occipitalis, cervical paraspinal, and trapezius). At the investigator's discretion, an additional $40 \mathrm{U}$ could be administered using a follow-thepain strategy. The primary endpoint was mean change from baseline in frequency of headache episodes for the 28 day period ending with Week 24. No significant between-group difference for onabotulinumtoxinA versus placebo was seen for this $(-5.2$ vs $-5.3 ; P=0.344)$. A high placebo response rate was observed. Significant between-group differences for onabotulinumtoxinA were observed for the secondary endpoints headache days $(P=0.006)$ and migraine days $(P=0.002)$ at all time points (including Week 24). The trial design of PREEMPT 2 was identical to its predecessor's and randomized 705 patients to either onabotulinumtoxinA or placebo. ${ }^{57}$ Similar to PREEMPT 1, most patients overused pain medications at baseline. The primary endpoint in PREEMPT 2 was the mean change from baseline in frequency of headache days for the 28 day period ending with Week 24. OnabotulinumtoxinA was significantly superior to placebo for this endpoint ( -9.0 headache days with onabotulinumtoxin $\mathrm{A}$ vs -6.7 placebo, $P<0.001$; mean intergroup difference -2.3 [95\% confidence interval $-3.25,-1.31]$ ).

Pooled analyses from PREEMPT 1 and 2 (1384 patients) demonstrated a mean decrease from baseline in frequency of headache days, with statistically significant between-group differences favoring onabotulinumtoxinA over placebo at Week $24(-8.4$ vs $-6.6 ; P<0.001)$ and at atl other time points. ${ }^{62}$ Significant differences favoring onabotulinumtoxinA were also observed for a number of secondary efficacy variables, including reductions in moderate or severe headache days $(P<0.001)$, cumulative hours of headache on headache days $(P<0.001)$, headache episodes $(P<0.009)$, the proportion of patients with a severe headache impact test- 6 score $(P<0.001)$, and migraine episodes $(P<0.004)$. 
To conclude, available randomized, double-blind, placebo-controlled trials suggest that onabotulinumtoxinA is effective in chronic migraine prophylaxis. ${ }^{53,62}$ It is important to note, however, that the therapeutic gain over placebo for many of the PREEMPT trials' outcomes was modest, even if statistically significant. ${ }^{56,57,62}$ As an example, in the pooled analysis, treatment with onabotulinumtoxinA led to a statistically significant reduction of headache days per 28 days compared with placebo ( 8.4 vs 6.6), but the absolute difference between the two groups was small (1.8 days); ${ }^{62}$ supporting the utility of onabotulinumtoxinA injection as moderately superior to placebo for the treatment of chronic migraine. This, the high cost of the toxin, and the need for an experienced clinician to administer onabotulinumtoxinA have led some authors to consider it as a second-line chronic migraine prophylactic therapy. ${ }^{63}$

Still, the excellent tolerability of onabotulinumtoxinA makes it an extremely attractive alternative for patients who fail to tolerate, and therefore discontinue, traditional oral prophylactics. In comparison with topiramate, for example, Mathew and Jaffri ${ }^{64}$ reported that patients receiving onabotulinumtoxinA had fewer adverse effects leading to discontinuation $(7.7 \%)$ than patients in the topiramate group $(24.1 \%)$. In another study, more than half of the study population had discontinued oral migraine prophylaxis within 3 months after commencing onabotulinumtoxinA. ${ }^{65}$ Because it is injected, compliance appears to be less of an issue when comparing onabotulinumtoxinA with oral prophylactics. ${ }^{62}$ OnabotulinumtoxinA may be a useful treatment option for headache patients demonstrating poor compliance, adherence, or adverse event profile with oral prophylactic regimens. ${ }^{66}$

\section{Unspecified migraine subtype or $\mathrm{CDH}$}

A small study looked at the effect of higher doses of onabotulinumtoxinA on migraine, ${ }^{52}$ without specifying episodic or chronic migraine. Forty-nine patients with more than five migraine attacks per month were randomized to receive either onabotulinumtoxinA or placebo into corrugator, frontalis, temporalis, sternocleidomastoid, occipitalis, and posterior neck muscles. The proportion of episodic versus chronic migraine patients is uncertain. The two standardized dosing schemes based on patient weight were $135 \mathrm{U}$ for those $<65 \mathrm{~kg}$ and $205 \mathrm{U}$ for those $\geq 65 \mathrm{~kg}$. The primary outcome measure was the average frequency of headache days measured during 30 day blocks for 3 months. The secondary outcome measure was the severity of attacks. No significant differences were observed between control and test groups at baseline on these measures. Importantly, a high dropout rate of 17 (34\%) led to a small sample size to be analyzed, which may have attenuated the power to detect any potential main effects of onabotulinumtoxinA in the prevention of migraine.

"Chronic daily headache" $(\mathrm{CDH})$ is a descriptive term, generally defined by $\geq 15$ headache days per month, ${ }^{67}$ that encompasses multiple headache diagnoses, including chronic migraine, chronic tension-type headache, new daily persistent headache, hemicrania continua, and others. Randomized, placebo-controlled studies evaluating onabotulinumtoxinA in $\mathrm{CDH}$ have yielded mixed results and are difficult to analyze, given the heterogeneity of the headache syndromes represented. For instance, a study by Ondo et al ${ }^{67}$ noted that only 14 of their 60 enrolled $\mathrm{CDH}$ subjects had chronic migraine, and the rest had chronic tension-type headache. This makes it difficult to draw strong conclusions from the study's somewhat positive results. ${ }^{60,68}$ Other investigators used onabotulinumtoxinA in a fixed-site approach for $\mathrm{CDH}$ prophylaxis in 702 enrolled patients, $53 \%$ of whom had a confirmed "transformed" (chronic) migraine diagnosis while the rest $(47 \%)$ had an unspecified or alternative $\mathrm{CDH}$ subtype. ${ }^{69}$ The primary efficacy endpoint (mean change from baseline in the frequency of headache-free days for the 30 day period ending on Day 180) was not met, although at Day 240 the decrease in headache frequency was significantly greater for the onabotulinumtoxinA $225 \mathrm{U}$ and 150 U groups compared with placebo. No subgroup statistical analysis was done based on $\mathrm{CDH}$ subtype. Another attempt to establish onabotulinumtoxinA as a probable effective $\mathrm{CDH}$ prophylactic using a follow-the-pain approach in a dose of 105-260 U failed to reach statistical significance for the primary efficacy measure (mean change from baseline in the frequency of headache-free days in a 30 day period), although a significantly higher percentage of onabotulinumtoxinA patients had $\mathrm{a} \geq 50 \%$ headache day frequency decrease from baseline per 30 day period at Day 180 (32.7\% vs $15.0 \%$, $P=0.027)$, the secondary efficacy measure. ${ }^{60}$ In this study, most patients $(61 \%$ of 355$)$ reported a history of migraine. However, precise $\mathrm{CDH}$ subtypes were not known.

\section{Safety and tolerability}

OnabotulinumtoxinA has been fairly well tolerated across studies. Reported adverse events with onabotulinumtoxinA in these randomized trials were transient, and were most frequently related to muscle weakness or pain at injections sites. Muscle weakness around the face and neck, for instance, has led to a higher number of patients in the onabotulinumtoxinA groups reporting neck pain, muscular weakness, eyelid 
ptosis, and diplopia. In the pooled analysis of the PREEMPT trials, only neck pain occurred, with an incidence of $>5 \%$ ( $6.7 \%$ onabotulinumtoxinA group vs $2.2 \%$ placebo group). The most frequently reported side effects leading to discontinuation of onabotulinumtoxinA injections were neck pain $(0.6 \%)$, muscular weakness $(0.4 \%)$, headache $(0.4 \%)$, and migraine $(0.4 \%){ }^{62}$

The safety of onabotulinumtoxinA in migraine management during pregnancy and lactation has not been established. Most randomized, double-blind, placebo-controlled studies using onabotulinumtoxinA in migraine prophylaxis have excluded pregnant and lactating women. With the exception of a woman who discontinued the study at Day 145 because of pregnancy and subsequently delivered a healthy child, ${ }^{49}$ no other pregnancy outcome has been reported in these trials. At present, onabotulinumtoxinA belongs to the pregnancy category $\mathrm{C}$ (risk cannot be ruled out) of the FDA and is not recommended during pregnancy. ${ }^{70}$

Outside of the studies included in this review, distant spread of onabotulinumtoxinA beyond the site of injection and systemic effects resembling botulism (dysphagia, breathing difficulties, generalized muscle weakness, among others) appear to be rare but have been reported even at low doses. ${ }^{71}$ Because of this, the FDA has required a boxed warning for all botulinum toxin products (see Figure 2).

\section{Disability and health-related quality of life}

It is well recognized that migraine attacks not only impair the individual's ability to function during the attack but also may affect an individual's functioning and overall well-being between attacks. ${ }^{1}$ Many of the randomized, controlled efficacy trials attempted to assess whether onabotulinumtoxin A treatment impacted the patients' migraine-related disability and health-related quality of life. The measuring instruments were not consistent throughout the efficacy studies, however, making comparison among them problematic. In some cases, the questionnaire used may not have been a validated tool or its source was not properly cited, further complicating the interpretation of their results.
Based on limited data available, there is some suggestion that onabotulinumtoxinA may improve the health-related quality of life in patients with chronic migraine. The PREEMPT trials looked at this using the Migraine-specific Quality of Life Questionnaire Version 2.1. Pooled analysis of the data from both trials showed that treatment with onabotulinumtoxinA significantly improved health-related quality of life $(P<0.001)$, as measured by changes from baseline in all three role function domains (restrictive, preventive, and emotional) at both time points evaluated (Weeks 12 and 24). ${ }^{62}$ Pooled analysis also demonstrated a clinically meaningful between-group difference for onabotulinumtoxinA versus placebo observed at Week 24 in mean change from baseline in total Headache Impact Test- 6 score (2.4; $P<0.001)$, a score that measures headache-related disability. Freitag et $\mathrm{al}^{53}$ also noted a positive trend when examining the effect of onabotulinumtoxinA on Migraine Disability Assessment Scores, but the differences did not meet statistical significance.

\section{Comparator studies}

Small studies comparing onabotulinumtoxinA with other first-line migraine preventives are promising. A randomized, double-blind study of 59 migraineurs showed that patients receiving onabotulinumtoxinA with an oral placebo had similar improvements in migraine disability scores, and similar reductions in headache days to patients receiving divalproex sodium and placebo injections. ${ }^{72}$ Another study of 72 patients showed that $250 \mathrm{U}$ of onabotulinumtoxinA had similar efficacy to $25 \mathrm{mg}$ or $50 \mathrm{mg}$ of amitriptyline for chronic migraine. ${ }^{73}$ Finally, two recent randomized, placebocontrolled trials (59 and 60 patients) compared up to $200 \mathrm{U}$ of onabotulinumtoxinA with topiramate and concluded that onabotulinumtoxinA had similar benefit to topiramate in the treatment of chronic migraine. ${ }^{64,74}$

\section{Dosing and administration}

Ample variation in onabotulinumtoxinA dose, injection sites, and strategy ("fixed-site, fixed-dose" or "followthe-pain") has been seen throughout the trials. The large

\section{WARNING: DISTANT SPREAD OF TOXIN EFFECT}

The effects of BOTOX and all botulinum toxin products may spread from the area of the injection to produce symptoms consistent with botulinum toxin effects. These symptoms have been reported hours to weeks after injection. Swallowing and breathing difficulties can be life threatening and there have been reports of death. The risk of symptoms is probably greatest in children treated for spasticity but symptoms can also occur in adults, particularly in those patients who have an underlying condition that would predispose them to these symptoms

Figure 2 US Food and Drug Administration box warning included on package insert for onabotulinumtoxinA (BOTOX ${ }^{\circledR}$ ). 


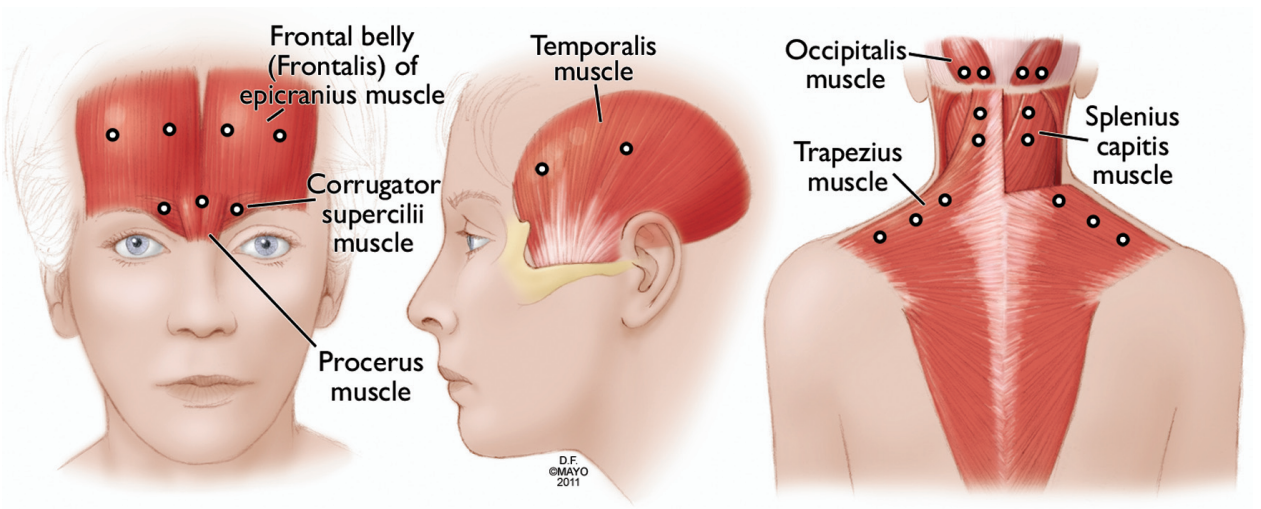

Figure 3 OnabotulinumtoxinA injection sites used by the authors (see Table 3 for dosing).

Note: Used with permission of Mayo Foundation for Medical Education and Research, all rights reserved.

multicenter PREEMPT trials used a fixed-site, fixed-dose injection paradigm, with additional follow-the-pain sites considered depending on individual symptoms. ${ }^{75}$ The PREEMPT paradigm has proven to be safe, well tolerated, and more effective than placebo, and has been recommended by some authors as the evidence-based approach to optimize clinical outcomes for patients with chronic migraine. ${ }^{56,57,75}$ Other authors have disputed the need for all of the injections sites included in the PREEMPT trials, and advocate further studies to identify the best injection strategy. ${ }^{76}$

Until future studies establish the optimal dose, injection sites, and protocol for onabotulinumtoxinA in chronic migraine prophylaxis, the standardized $155 \mathrm{U}$ injection protocol tested in the PREEMPT trials is a great and welcomed advance. Unfortunately, the recommended $155 \mathrm{U}$ dose creates obvious problems in resource utilization and cost effectiveness. In the US, onabotulinumtoxinA is available in single-use $50 \mathrm{U}, 100 \mathrm{U}$, or $200 \mathrm{U}$ vials. If one were to routinely

Table 3 Standard fixed-site, fixed-dose $150 \cup$ protocol for onabotulinumtoxinA currently used by the authors at the time of this manuscript's publication

- $5.0 \mathrm{U}$ into each corrugator muscle and into the procerus muscle One injection site per muscle ( $15.0 \mathrm{U}$ total)

- $5.0 \mathrm{U}$ into the right and left superior frontalis muscle Two injection sites per muscle ( $10 \mathrm{U}$ total)

- $12.5 \mathrm{U}$ into each temporalis muscle Two injection sites per muscle ( $25 \mathrm{U}$ total)

- $12.5 \mathrm{U}$ into each splenius capitis muscle

Two injection sites per muscle administered as two-thirds of I2.5 $U(8.3 \mathrm{U})$ at superior injection site (near muscle insertion) and one third $(4.2 \mathrm{U})$ at mid-belly of muscle to minimize neck weakness (25 U total)

- $\quad 12.5 \mathrm{U}$ into each occipitalis muscle

Two injection sites per muscle ( $25 \mathrm{U}$ total)

- $25 \mathrm{U}$ into each trapezius muscle Three injection sites per muscle ( $50 \mathrm{U}$ total) use the $155 \mathrm{U}$ protocol, this could lead to a significant waste of nonreusable product.

At our institution, most chronic migraine patients get a $150 \mathrm{U}$ protocol (Figure 3, Table 3) in a fixed-site, fixeddose fashion targeting similar injection sites to those in the PREEMPT injection protocol. This is occasionally combined with an additional follow-the-pain site on an individual case basis. There is no indisputable evidence yet supporting that adding the follow-the-pain strategy is superior to the $155 \mathrm{U}$ fixed-site, fixed-dose protocol alone. OnabotulinumtoxinA injections are administered typically every 12 weeks. More frequent administration is avoided to minimize the formation of neutralizing antibodies, which can affect onabotulinumtoxinA efficacy. ${ }^{26}$

\section{Conclusion}

Based on the available data, onabotulinumtoxinA has not been convincingly shown to be effective in the prevention of episodic migraine. In chronic migraine prophylaxis, however, available randomized, double-blind, placebo-controlled trials suggest that onabotulinumtoxinA is effective. The injected form and excellent tolerability of onabotulinumtoxinA makes it an extremely attractive alternative for chronic migraine patients who have demonstrated poor tolerance or poor compliance with traditional oral prophylactics. Although the mechanisms through which onabotulinumtoxinA may exert its benefit remain uncertain, onabotulinumtoxinA is a welcome addition to the available treatment options for chronic migraine, which is often a disabling and difficultto-manage condition.

\section{Disclosure}

Carrie E Robertson has received honoraria for continuing education courses related to neuroimaging. She reports no conflicts of interest. Ivan Garza receives compensation as 
an author for Up-to-Date and as headache section editor for Current Neurology and Neuroscience Reports. He reports no conflicts of interest.

\section{References}

1. Lipton RB, Bigal ME. Migraine: epidemiology, impact, and risk factors for progression. Headache. 2005;(45 suppl 1):S3-S13.

2. IHS. The International Classification of Headache Disorders: 2nd edition. Cephalalgia. 2004;(24 suppl 1):9-160.

3. Olesen J, Bousser MG, Diener HC, et al. New appendix criteria open for a broader concept of chronic migraine. Cephalalgia. 2006;26(6): 742-746.

4. Manack AN, Buse DC, Lipton RB. Chronic migraine: epidemiology and disease burden. Curr Pain Headache Rep. 2011;15(1):70-78.

5. Silberstein SD. Treatment recommendations for migraine. Nat Clin Pract Neurol. 2008;4(9):482-489.

6. Binder WJ, Brin MF, Blitzer A, Pogoda JM. Botulinum toxin type A (BOTOX) for treatment of migraine. Semin Cutan Med Surg. 2001;20(2):93-100.

7. Binder WJ, Brin MF, Blitzer A, et al. Botulinum toxin type A (BOTOX) for treatment of migraine headaches: an open-label study. Otolaryngol Head Neck Surg. 2000;123(6):669-676.

8. Binder WJ, Blitzer A, Brin MF. Treatment of hyperfunctional lines of the face with botulinum toxin A. Dermatol Surg. 1998;24(11): 1198-1205.

9. Dressler D, Benecke R. Pharmacology of therapeutic botulinum toxin preparations. Disabil Rehabil. 2007;29(23):1761-1768.

10. Aktories K, Barmann M, Ohishi I, et al. Botulinum C2 toxin ADPribosylates actin. Nature. 1986;322(6077):390-392.

11. Bhidayasiri R, Truong DD. Expanding use of botulinum toxin. J Neurol Sci. 15 2005;235(1-2):1-9.

12. Sampaio C, Costa J, Ferreira JJ. Clinical comparability of marketed formulations of botulinum toxin. Mov Disord. 2004;(19 suppl 8): S129-S136.

13. FDA U. FDA gives update on botulinum toxin safety warnings; established names of drugs changed: FDA News Release.

14. Albanese A. Terminology for preparations of botulinum neurotoxins: what a difference a name makes. Jama. 2011;305(1):89-90.

15. Frevert J, Dressler D. Complexing proteins in botulinum toxin type $\mathrm{A}$ drugs: a help or a hindrance? Biologics. 2010;4:325-332.

16. Foran PG, Mohammed N, Lisk GO, et al. Evaluation of the therapeutic usefulness of botulinum neurotoxin $\mathrm{B}, \mathrm{C} 1, \mathrm{E}$, and $\mathrm{F}$ compared with the long lasting type A. Basis for distinct durations of inhibition of exocytosis in central neurons. J Biol Chem. 2003;278(2): 1363-1371.

17. Rosales RL, Bigalke H, Dressler D. Pharmacology of botulinum toxin: differences between type A preparations. Eur J Neurol. 2006 (13 suppl 1):2-10.

18. Dolly JO, Lawrence GW, Meng J, et al. Neuro-exocytosis: botulinum toxins as inhibitory probes and versatile therapeutics. Curr Opin Pharmacol. 2009;9(3):326-335.

19. Brin MF, Fahn S, Moskowitz C, et al. Localized injections of botulinum toxin for the treatment of focal dystonia and hemifacial spasm. $A d v$ Neurol. 1988;50:599-608.

20. Eleopra R, Tugnoli V, De Grandis D. The variability in the clinical effect induced by botulinum toxin type A: the role of muscle activity in humans. Mov Disord. 1997;12(1):89-94.

21. Frasson E, Priori A, Ruzzante B, et al. Nerve stimulation boosts botulinum toxin action in spasticity. Mov Disord. 2005;20(5):624-629.

22. Wey JJ, Tang SS, Wu TY. Disulfide bond reduction corresponds to dimerization and hydrophobi-city changes of Clostridium botulinum type A neurotoxin. Acta Pharmacol Sin. 2006;27(9):1238-1246.

23. Foran PG, Davletov B, Meunier FA. Getting muscles moving again after botulinum toxin: novel therapeutic challenges. Trends Mol Med. 2003;9(7):291-299.
24. Dolly JO, Aoki KR. The structure and mode of action of different botulinum toxins. Eur J Neurol. 2006;(13 suppl 4):1-9.

25. Meunier FA, Schiavo G, Molgo J. Botulinum neurotoxins: from paralysis to recovery of functional neuromuscular transmission. J Physiol Paris. 2002;96(1-2):105-113.

26. Brin MF. Botulinum toxin: chemistry, pharmacology, toxicity, and immunology. Muscle Nerve Suppl. 1997;6:S146-S168.

27. Aoki KR. Review of a proposed mechanism for the antinociceptive action of botulinum toxin type A. Neurotoxicology. 2005;26(5):785-793.

28. Pavone F, Luvisetto S. Botulinum neurotoxin for pain management: insights from animal models. Toxins. 2010;2:2890-2913.

29. Mense $\mathrm{S}$. Neurobiological basis for the use of botulinum toxin in pain therapy. J Neurol. 2004;(251 suppl 1):I1-I7.

30. Arezzo JC. Possible mechanisms for the effects of botulinum toxin on pain. Clin J Pain. 2002;18(6 suppl):S125-S132.

31. Welch MJ, Purkiss JR, Foster KA. Sensitivity of embryonic rat dorsal root ganglia neurons to Clostridium botulinum neurotoxins. Toxicon. 2000;38(2):245-258.

32. Durham PL, Cady R. Regulation of calcitonin gene-related peptide secretion from trigeminal nerve cells by botulinum toxin type A: implications for migraine therapy. Headache. 2004;44(1):35-42; discussion 42-33.

33. Cui M, Khanijou S, Rubino J, Aoki KR. Subcutaneous administration of botulinum toxin A reduces formalin-induced pain. Pain. Jan 2004; 107(1-2):125-133.

34. Blersch W, Schulte-Mattler WJ, Przywara S, et al. Botulinum toxin A and the cutaneous nociception in humans: a prospective, double-blind, placebo-controlled, randomized study. J Neurol Sci. 2002;205(1):59-63.

35. Bach-Rojecky L, Lackovic Z. Antinociceptive effect of botulinum toxin type a in rat model of carrageenan and capsaicin induced pain. Croat Med J. 2005;46(2):201-208.

36. Bach-Rojecky L, Relja M, Lackovic Z. Botulinum toxin type A in experimental neuropathic pain. J Neural Transm. 2005;112(2):215-219.

37. Marinelli S, Luvisetto S, Cobianchi S, et al. Botulinum neurotoxin type A counteracts neuropathic pain and facilitates functional recovery after peripheral nerve injury in animal models. Neuroscience. 2010;171(1): 316-328.

38. Mika J, Rojewska E, Makuch W, et al. The effect of botulinum neurotoxin A on sciatic nerve injury-induced neuroimmunological changes in rat dorsal root ganglia and spinal cord. Neuroscience. 2011;175: $358-366$.

39. Ranoux D, Attal N, Morain F, Bouhassira D. Botulinum toxin type A induces direct analgesic effects in chronic neuropathic pain. Ann Neurol. 2008;64(3):274-283.

40. Favre-Guilmard C, Auguet M, Chabrier PE. Different antinociceptive effects of botulinum toxin type A in inflammatory and peripheral polyneuropathic rat models. Eur J Pharmacol. 2009;617(1-3):48-53.

41. Bach-Rojecky L, Salkovic-Petrisic M, Lackovic Z. Botulinum toxin type A reduces pain supersensitivity in experimental diabetic neuropathy: bilateral effect after unilateral injection. Eur J Pharmacol. 2010;633(1-3):10-14

42. Caleo M, Antonucci F, Restani L, Mazzocchio R. A reappraisal of the central effects of botulinum neurotoxin type A: by what mechanism? J Neurochem. 2009;109(1):15-24.

43. Kitamura Y, Matsuka Y, Spigelman I, et al. Botulinum toxin type a $(150 \mathrm{kDa})$ decreases exaggerated neurotransmitter release from trigeminal ganglion neurons and relieves neuropathy behaviors induced by infraorbital nerve constriction. Neuroscience. 2009;159(4): $1422-1429$.

44. Caleo M, Schiavo G. Central effects of tetanus and botulinum neurotoxins. Toxicon. Oct 2009;54(5):593-599.

45. Antonucci F, Rossi C, Gianfranceschi L, et al. Long-distance retrograde effects of botulinum neurotoxin A. $J$ Neurosci. 2008;28(14): 3689-3696.

46. Matak I, Bach-Rojecky L, Filipovic B, Lackovic Z. Behavioral and immunohistochemical evidence for central antinociceptive activity of botulinum toxin A. Neuroscience. 2011;186:201-207. 
47. Silberstein S, Mathew N, Saper J, Jenkins S. Botulinum toxin type A as a migraine preventive treatment. For the BOTOX Migraine Clinical Research Group. Headache. 2000;40(6):445-450.

48. Evers S, Vollmer-Haase J, Schwaag S, et al. Botulinum toxin A in the prophylactic treatment of migraine - a randomized, double-blind, placebo-controlled study. Cephalalgia. 2004;24(10):838-843.

49. Elkind AH, O'Carroll P, Blumenfeld A, et al. A series of three sequential, randomized, controlled studies of repeated treatments with botulinum toxin type A for migraine prophylaxis. J Pain. 2006;7(10):688-696.

50. Aurora SK, Gawel M, Brandes JL, et al. Botulinum toxin type a prophylactic treatment of episodic migraine: a randomized, doubleblind, placebo-controlled exploratory study. Headache. 2007;47(4): 486-499.

51. Anand KS, Prasad A, Singh MM, et al. Botulinum toxin type A in prophylactic treatment of migraine. Am J Ther. 2006;13(3): 183-187.

52. Vo AH, Satori R, Jabbari B, et al. Botulinum toxin type-a in the prevention of migraine: a double-blind controlled trial. Aviat Space Environ Med. 2007;78(5 suppl):B113-B118.

53. Freitag FG, Diamond S, Diamond M, Urban G. Botulinum toxin type $\mathrm{A}$ in the treatment of chronic migraine without medication overuse. Headache. 2008;48(2):201-209.

54. Relja M, Poole AC, Schoenen J, et al. A multicentre, double-blind, randomized, placebo-controlled, parallel group study of multiple treatments of botulinum toxin type A (BoNTA) for the prophylaxis of episodic migraine headaches. Cephalalgia. 2007;27(6): 492-503.

55. Saper JR, Mathew NT, Loder EW, et al. A double-blind, randomized, placebo-controlled comparison of botulinum toxin type a injection sites and doses in the prevention of episodic migraine. Pain Med. 2007;8(6): 478-485.

56. Aurora SK, Dodick DW, Turkel CC, et al. OnabotulinumtoxinA for treatment of chronic migraine: results from the double-blind, randomized, placebo-controlled phase of the PREEMPT 1 trial. Cephalalgia. 2010;30(7):793-803.

57. Diener HC, Dodick DW, Aurora SK, et al. OnabotulinumtoxinA for treatment of chronic migraine: results from the double-blind, randomized, placebo-controlled phase of the PREEMPT 2 trial. Cephalalgia. 2010;30(7):804-814.

58. Chan VW, McCabe EJ, MacGregor DL. Botox treatment for migraine and chronic daily headache in adolescents. J Neurosci Nurs. 2009;41(5): 235-243.

59. Ahmed K, Oas KH, Mack KJ, Garza I. Experience with botulinum toxin type A in medically intractable pediatric chronic daily headache. Pediatr Neurol. 2010;43(5):316-319.

60. Mathew NT, Frishberg BM, Gawel M, et al. Botulinum toxin type A (BOTOX) for the prophylactic treatment of chronic daily headache: a randomized, double-blind, placebo-controlled trial. Headache. 2005;45(4):293-307.
61. Naumann M, So Y, Argoff CE, et al. Assessment: botulinum neurotoxin in the treatment of autonomic disorders and pain (an evidence-based review): report of the Therapeutics and Technology Assessment Subcommittee of the American Academy of Neurology. Neurology. 2008;70(19):1707-1714.

62. Dodick DW, Turkel CC, Degryse RE, et al. OnabotulinumtoxinA for treatment of chronic migraine: pooled results from the double-blind, randomized, placebo-controlled phases of the PREEMPT clinical program. Headache. 2010;50(6):92-136.

63. Garza I, Schwedt TJ. Chronic migraine. UpToDate, Wolters Kluwer Health. 2011;Desktop 19.1. Accessed June 18, 2011.

64. Mathew NT, Jaffri SF. A double-blind comparison of onabotulinumtoxina (BOTOX) and topiramate (TOPAMAX) for the prophylactic treatment of chronic migraine: a pilot study. Headache. 2009;49(10):1466-1478.

65. Rahimtoola H, Buurma H, Tijssen CC, et al. Migraine prophylactic medication usage patterns in The Netherlands. Cephalalgia. 2003;23(4):293-301.

66. Cady R, Schreiber C. Botulinum toxin type A as migraine preventive treatment in patients previously failing oral prophylactic treatment due to compliance issues. Headache. 2008;48(6):900-913.

67. Ondo WG, Vuong KD, Derman HS. Botulinum toxin A for chronic daily headache: a randomized, placebo-controlled, parallel design study. Cephalalgia. 2004;24(1):60-65.

68. Silberstein SD, Stark SR, Lucas SM, et al. Botulinum toxin type A for the prophylactic treatment of chronic daily headache: a randomized, double-blind, placebo-controlled trial. Mayo Clin Proc. 2005;80(9):1126-1137.

69. Silberstein SD, Stark SR, Lucas SM, et al. Botulinum toxin type A for the prophylactic treatment of chronic daily headache: a randomized, double-blind, placebo-controlled trial. Mayo Clinic proceedings. Mayo Clinic. Sep 2005;80(9):1126-1137.

70. Allergan. Botox/Botox Cosmetic package insert:17.

71. Woodcock J. Response to citizen petition on botulinum toxin (Docket No. FDA-2008-P-0061). In: FDA, ed 2009.

72. Blumenfeld AM, Schim JD, Chippendale TJ. Botulinum toxin type A and divalproex sodium for prophylactic treatment of episodic or chronic migraine. Headache. 2008;48(2):210-220.

73. Magalhaes E, Menezes C, Cardeal M, Melo A. Botulinum toxin type A versus amitriptyline for the treatment of chronic daily migraine. Clin Neurol Neurosurg. 2010;112(6):463-466.

74. Cady RK, Schreiber CP, Porter JA, et al. A multi-center double-blind pilot comparison of onabotulinumtoxinA and topiramate for the prophylactic treatment of chronic migraine. Headache. 2011;51(1):21-32.

75. Blumenfeld A, Silberstein SD, Dodick DW, et al. Method of injection of onabotulinumtoxinA for chronic migraine: a safe, well-tolerated, and effective treatment paradigm based on the PREEMPT clinical program. Headache. 2010;50(9):1406-1418.

76. Gerwin R. Treatment of chronic migraine headache with onabotulinumtoxinA. Curr Pain Headache Rep. 2011;15(5):336-338.
Neuropsychiatric Disease and Treatment

\section{Publish your work in this journal}

Neuropsychiatric Disease and Treatment is an international, peerreviewed journal of clinical therapeutics and pharmacology focusing on concise rapid reporting of clinical or pre-clinical studies on a range of neuropsychiatric and neurological disorders. This journal is indexed on PubMed Central, the 'PsycINFO' database and CAS.

\section{Dovepress}

The manuscript management system is completely online and includes a very quick and fair peer-review system, which is all easy to use. Visit http://www.dovepress.com/testimonials.php to read real quotes from published authors. 